\title{
Different cambial activities in response to climatic factors of three Malaysian rainforest Shorea species with different stem diameters
}

\begin{abstract}
We investigated the cambial activities in Shorea leprosula, Shorea acuminata, and Shorea parvifolia trees with different stem diameters at breast height growing in a natural lowland dipterocarp rainforest in Malaysia. We collected and analyzed samples from the main trunks of selected trees in monthly intervals from March 2013 to April 2014. The cambium of the three Shorea species showed periods of active and less active growth in the humid forest. Selected trees with different stem diameters of the same and different species showed variation in rhythms of cambial activity (changes in the numbers of cambial and enlarging cell layers) at site with similar growing conditions. Monthly mean relative humidity and monthly mean vapour pressure deficit were important factors influencing the number of cambial cells of $S$. acuminata with larger stem diameter, and those of $S$. parvifolia with different stem diameters. Active cambium were detected in months with high relative humidity and low vapour pressure deficit in those trees. Total monthly precipitation had significant positive correlation with the cambial cells activities of $S$. parvifolia with different stem diameters, but no correlation with those of the other two species. The cambium of $S$. leprosula with smaller and larger stem diameters were found active throughout the year. We concluded that the three Shorea species examined with different stem diameters have different growth strategies and thus react differently to climatic factors.
\end{abstract}

Keyword: Cambial activity; Shorea species; Stem diameter; Tropical rainforest 